\title{
Effect of Codon Optimisation on the Production of Recombinant Fish Growth Hormone in Pichia pastoris
}

\author{
Hussin A. Rothan, ${ }^{1}$ Teh Ser Huy, ${ }^{2}$ and Zulqarnain Mohamed $^{2}$ \\ ${ }^{1}$ Institute of Research Management \& Monitoring, Deputy Vice Chancellor (Research \& Innovation) Building, \\ University of Malaya, 50603 Kuala Lumpur, Malaysia \\ ${ }^{2}$ Genetics and Molecular Biology Unit, Institute of Biological Sciences, Faculty of Science, University of Malaya, \\ 50603 Kuala Lumpur, Malaysia
}

Correspondence should be addressed to Zulqarnain Mohamed; zulq@um.edu.my

Received 24 April 2014; Accepted 9 July 2014; Published 22 July 2014

Academic Editor: Héla Kallel

Copyright (C) 2014 Hussin A. Rothan et al. This is an open access article distributed under the Creative Commons Attribution License, which permits unrestricted use, distribution, and reproduction in any medium, provided the original work is properly cited.

\begin{abstract}
This study was established to test the hypothesis of whether the codon optimization of fish growth hormone gene (FGH) based on $P$. pastoris preferred codon will improve the quantity of secreted $\mathrm{rFGH}$ in culture supernatant that can directly be used as fish feed supplements. The optimized $F G H$ coding sequence $(o F G H)$ and native sequence $(n F G H)$ of giant grouper fish (Epinephelus lanceolatus) were cloned into $P$. pastoris expression vector (pPICZ $\alpha \mathrm{A}$ ) downstream of alcohol oxidase gene (AOX1) for efficient induction of extracellular $\mathrm{rFGH}$ by adding $1 \%$ of absolute methanol. The results showed that recombinant $P$. pastoris was able to produce $2.80 \pm 0.27 \mathrm{mg}$ of oFGH compared to $1.75 \pm 0.25$ of nFGH in one litre of culture supernatant. The total body weight of tiger grouper fingerlings fed with oFGH increased significantly at third $(P<0.05)$ and fourth weeks $(P<0.01)$ of fourweek experiment period compared to those fed with $\mathrm{nFGH}$. Both oFGH and nFGH significantly enhanced the final biomass and fish survival percentage. In conclusion, codon optimization of $F G H$ fragment was useful to increase rFGH quantity in the culture supernatant of $P$. pastoris that can be directly used as fish feed supplements. Further studies are still required for large scale production of $\mathrm{rFGH}$ and practical application in aquaculture production.
\end{abstract}

\section{Introduction}

Fish growth hormone (FGH), a protein hormone with molecular weight of $22 \mathrm{kDa}$, is produced by somatotroph cells in the anterior pituitary gland of fish. It regulates growth and development in fish $[1,2]$. Using recombinant FGH as a supplement in the feed, fish growth rate was increased without accumulation of FGH in the fish body $[3,4]$. In aquaculture applications, fish growth rate has been increased after using recombinant FGH expressed in E. coli [5]. However, the low capacity of posttranslation process in E. coli resulted in less active recombinant proteins and formation of insoluble inclusion bodies [6]. Insoluble inclusion bodies require more steps in protein purification process and a complicated procedure for protein refolding to recover its biological function. It has also been known that $E$. coli is a prokaryote and its intrinsic characteristics differ from those of eukaryotes, such as protein processing, protein folding, and posttranslational modifications [7]. Fish growth hormone has been expressed in the yeast Saccharomyces cerevisiae [8-10] and the yeast Pichia pastoris [11]. The yeast $P$. pastoris expression system offers advantages over $S$. cerevisiae in its high productivity, efficient secreted expression, and stable genetics, so it has been an attractive candidate for production of foreign proteins [12]. Intracellular expression of FGH in $P$. pastoris that is used as feed supplement showed significant increase in growth rate on tilapia [13], but the expression of recombinant FGH was low (1-2\% of the total cellular proteins). Interestingly, $P$. pastoris has a higher secretory capacity and lower expression level of endogenous proteins than other yeasts. Recombinant proteins comprise the majority of the total secreted proteins in the medium [14]. Of note, fish growth hormone cDNA was used in most of previous studies to produce recombinant FGH in different 
expression systems [5, 8-11]. In our study, we constructed a synthetic FGH gene with preferred codons of $P$. pastoris in order to increase the expression level of recombinant FGH. Additionally, producing extracellular FGH can omit a purification process and reduce the cost of production in fish farming.

\section{Materials and Methods}

2.1. Culture Media. For cloning purposes, E. coli strain TOP10 was cultured in low salt LB medium (LSLB) and LSLBagar with Zeocin (salt concentration $<90 \mathrm{mM}$, pH 7.5 for Zeocin to be active). The solid medium (LSLB-agar) contains $1 \%$ peptone, $0.05 \% \mathrm{NaCl}, 0.5 \%$ yeast extract, and $1.5 \%$ agar with $25 \mu \mathrm{g} / \mathrm{mL}$ Zeocin and the liquid medium was YPD broth, containing $2 \%$ peptone, $1 \%$ yeast extract, $2 \%$ dextrose, and $100 \mathrm{mg} / \mathrm{L}$ Zeocin. P. pastoris was cultured on YPDSagar containing $2 \%$ agar and $18 \%$ sorbitol. For expression purposes, the buffered complex media, BMMY and BMGY, containing $2 \%$ peptone, $1 \%$ yeast extract, $4 \times 10^{-5} \%$ biotin, $1.34 \%$ yeast nitrogen base, $0.1 \mathrm{M}$ potassium phosphate buffer (pH 6.0), and 1\% glycerol (for BMGY growth medium) or $1 \%$ methanol (for BMMY induction medium) were used.

2.2. DNA Preparation of FGH Fragments. Total RNA was isolated from the pituitary glands of giant grouper fish (Epinephelus lanceolatus) using RNA extraction kit (Invitogen, The Netherlands). The native FGH ( $n F G H)$ gene was reverse-transcripted and amplified using reverse transcriptase kit (Invitrogen, The Netherlands). The $n F G H$ gene fragment was cloned into pGEM-T cloning vector (Promega, USA) and transformed into E. coli strain JM109. DNA sequencing was performed and the sequence was compared with NCBI database (http://www.ncbi.nlm.nih.gov/) for verification.

The codon-optimized FGH (oFGH) sequence was synthesised according to the $P$. pastoris preferred codons by Invitrogen (http://www.invitrogen.com/genesynthesis). Depending on synthesiser information, the following sequence regions were avoided or amended: (i) very high $(>80 \%)$ or very low $(<30 \%)$ GC content, (ii) the cis-acting sequence motifs, such as internal TATA-boxes, chi-sites, and ribosomal entry sites, and (iii) AT-rich or GC-rich sequence that stretches RNA instability motifs repeat sequences and RNA secondary structures splice donor and acceptor sites in higher eukaryotes.

2.3. Construction of Expression Vectors, $p P I C Z \alpha A-n F G H$ and $p P I C Z \alpha A-o F G H$. Construction of recombinant PICZ $\alpha A-$ $n F G H$ and PICZ $\alpha$ A-oFGH was carried out as descried previously [15]. In brief, the pGEM-T cloning vectors containing $n F G H$ or $o F G H$ gene fragments were digested with EcoRI and NotI restriction enzymes and the fragments with molecular weight of about $600 \mathrm{bp}$ were purified using QIAquick Gel Extraction kit (Qiagen, USA). The purified fragments were cloned separately into a EcoRI- and NotI-digested pPICZ $\alpha \mathrm{A}$ vector. The recombinant plasmids, pPICZ $\alpha \mathrm{A}$ $n F G H$ and pPICZ $\alpha \mathrm{A}-o F G H$, were transformed into E. coli strain Top10 of for propagation purpose. These recombinant plasmids were then isolated, sequenced, and linearized with SacI restriction enzyme. Subsequently, linearized pPICZ $\alpha$ A$n F G H$ and pPICZ $\alpha \mathrm{A}-o F G H$ were introduced into P. pastoris, wild-type X-33 strain using EasySelect Pichia Expression kit (Invitrogen, The Netherlands).

\section{Expression in $P$. pastoris}

Production of recombinant FGH in $P$. pastoris was carried out as described previously [15]. In brief, a single colony of recombinant X-33 harbouring $\mathrm{nFGH}$ and oFGH was inoculated in BMGY medium, respectively, and grown at $30^{\circ} \mathrm{C}$ until $\mathrm{OD}_{600}$ was 2-6. Cell pellet was collected by centrifugation and resuspended in BMMY media (or BGMY medium for control culture) at $\mathrm{OD}_{600}=1$. Incubation was continued at $30^{\circ} \mathrm{C}$ with shaking at $220 \mathrm{rpm}$. Methanol was added into BMMY medium to a final concentration of $0.5 \%$ for 12 -hour intervals while glycerol was added to the BMGY medium as a substitute for methanol. Culture supernatants were harvested at $24,36,48,60$, and 72 hours after induction and analysed by native SDS-PAGE and SDS-PAGE under denaturing and nondenaturing conditions as described previously [15]. The recombinant protein concentrations in culture supernatant were determined using fish growth hormone, FGH ELISA kit (Cat. no. E0044f, China, http://www.eiaab.com/).

3.1. Bioactivity Test. Tiger grouper fingerlings were divided randomly into four groups, 20 per group (control 1 was fed with common fish feed; control 2 was fed with common feed mixed with 5\% culture supernatant from wild-type P. pastoris; treatment 1 was fed with common feed mixed with 5\% culture supernatant from recombinant $P$. pastoris producing $\mathrm{nFGH}$; treatment 2 was fed with common feed mixed with 5\% culture supernatant from recombinant $P$. pastoris producing oFGH). The average body weight of fingerlings was approximately $10.5 \mathrm{~g}$ and the average body length was $8.5 \mathrm{~cm}$. Tanks and air stones were cleaned, disinfected, and refilled with new treated fresh water. New treated fresh water was replaced every 7 days for 31 days of experiment period. Feed distribution was done three times per day: 9:00 a.m., 1:00 p.m., and 6:00 p.m. Sampling was carried out every seven days. The supernatant of recombinant yeast culture was mixed with fish feed by $5 \%$ of total feed weight.

\section{Results and Discussion}

Purification and administration methods of recombinant fish growth hormone (FGH) are the main concerns of impracticable use in mass-scale aquaculture. Therefore, we conducted this study to test the hypothesis whether the optimized DNA sequence of FGH will significantly enhance its expression level in P. pastoris in order to be used as fish feed supplement. The entire FGH coding sequence was constructed based on $P$. pastoris preferred codons (oFGH) while the native FGH $(n F G H)$ gene was obtained by reverse transcription of total RNA that was extracted from fish pituitary gland (Figure 1). The pPICZ $\alpha \mathrm{A}$ plasmid contains Saccharomyces 


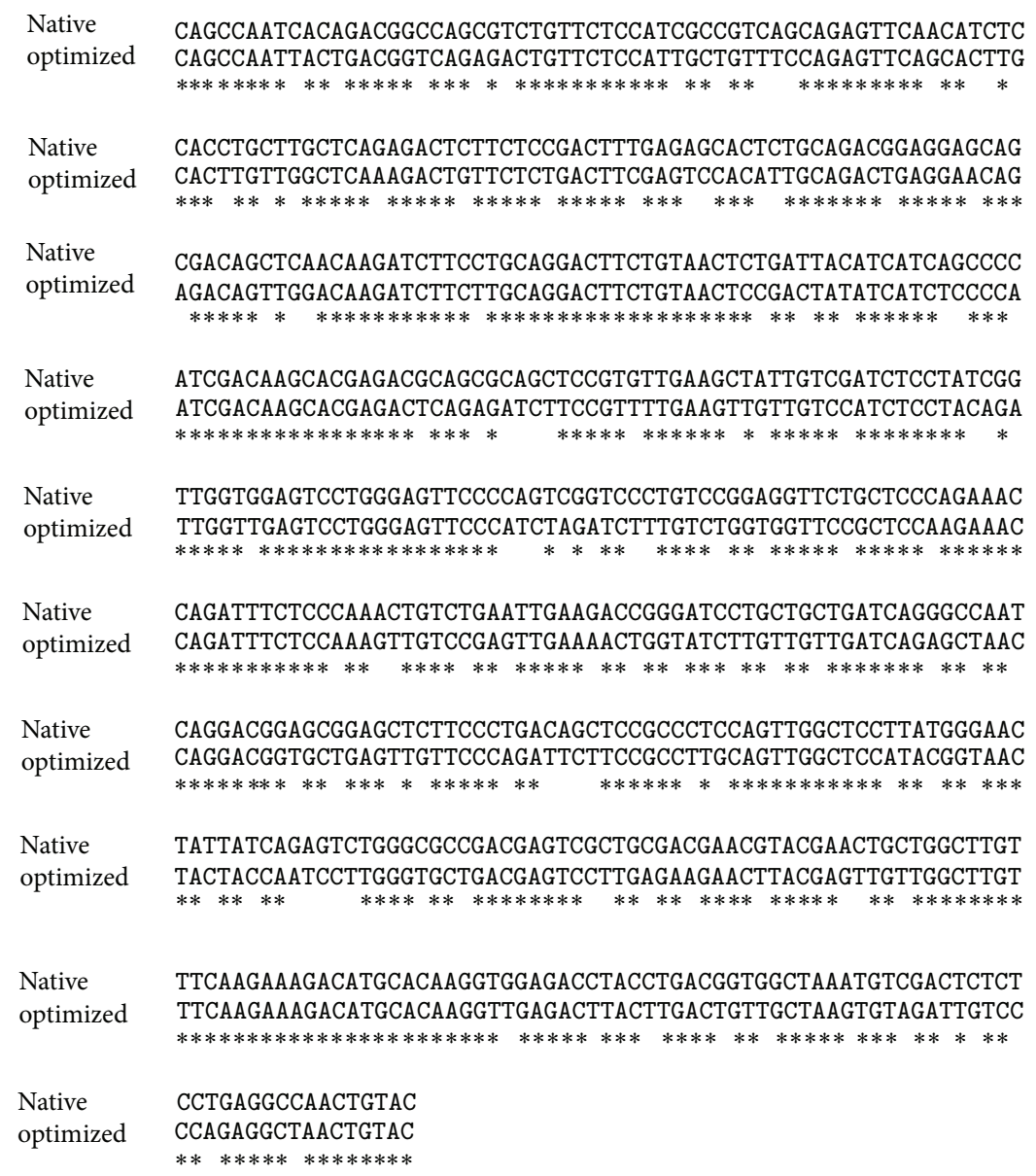

FIGURE 1: Sequences aligmnent of native fish growth hormone (nFGH) and optimized sequence based on P. pastoris preferred codons.

cerevisiae $\alpha$-factor secretion signal peptide for extracellular protein secretion. Both DNA fragments were cloned into $P$. pastoris expression vector ( $\mathrm{pPICZ} \alpha \mathrm{A})$ downstream $\alpha$-factor secretion signal peptide and the promoter of alcohol oxidase gene $(A O X 1)$ for efficient induction of extracellular $\mathrm{FGH}$ production by adding $1 \%$ of absolute methanol.

The results showed that the expression of $\mathrm{nFGH}$ and oFGH were detected by SDS-PAGE under denaturing conditions at the expected size of $22 \mathrm{kDa}$ (Figure 2). Further analysis by SDS-PAGE under denaturing and nondenaturing conditions showed that both FGH forms ( $\mathrm{nFGH}$ and oFGH) were produced by $P$. pastoris as monomers and multimers (Figure 3). After induction of recombinant yeast with methanol, the yeast was grown for $72 \mathrm{hrs}$, and then the production of FGH was quantified by FGH ELISA assay using standard FGH as a reference. The results showed that recombinant $P$. pastoris was able to produce $2.80 \pm 0.27 \mathrm{mg}$ of oFGH compared to $1.75 \pm 0.25 \mathrm{mg}$ of $\mathrm{nFGH}$ in one litre of culture supernatant (Figure 4).

In this study, $P$. pastoris was more efficient in producing oFGH compared to $\mathrm{nFGH}$ as secreted protein. It has been shown that codon optimization is important to increase translation rate through the direct use of host cell tRNA

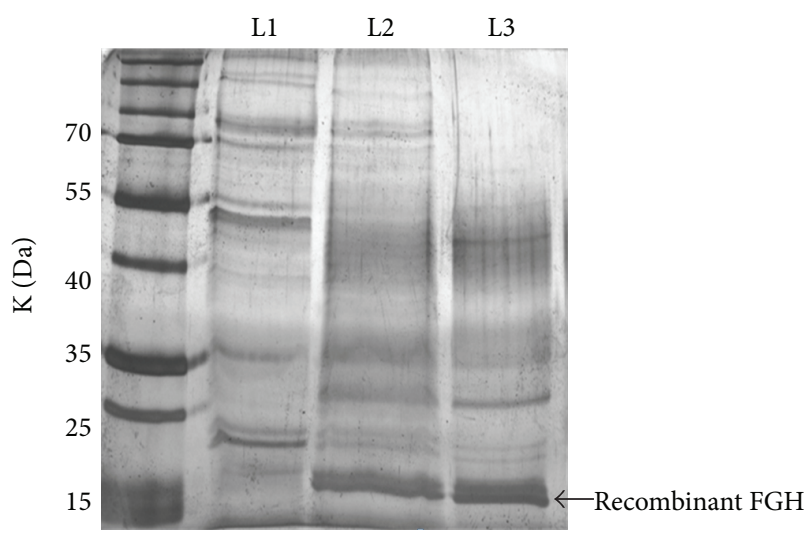

FIGURE 2: Production of native FGH (nFGH) and optimized FGH (oFGH) in $P$. pastoris. The expression vectors that contain oFGH and $\mathrm{nFGH}$ were transformed separately into Pichia pastoris strain X-33 using EasySelect Pichia Expression kit. A pilot experiment was carried out to identify the ability of $P$. pastoris to produce recombinant $\mathrm{FGH}$ as an extracellular protein. The SDS-PAGE result showed that the recombinant $\mathrm{nFGH}$ and $\mathrm{oFGH}$ were produced by P. pastoris at expected size of $22 \mathrm{kDa}$. $\mathrm{L} 1=$ control; $\mathrm{L} 2=\mathrm{nFGH}$; $\mathrm{L} 3=$ oFGH. 


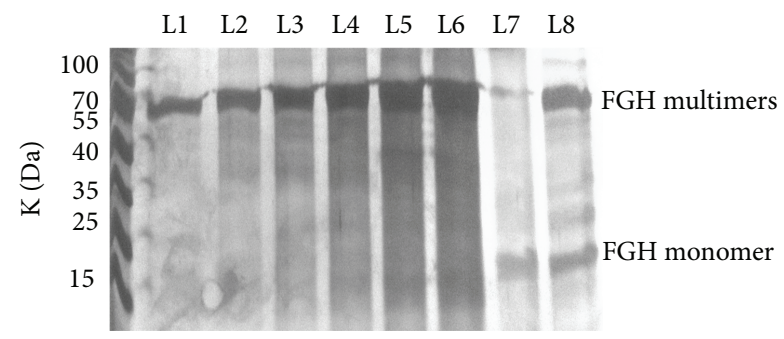

FIGURE 3: SDS-PAGE analysis of oFGH under denaturing and nondenaturing conditions. Recombinant $P$. pastoris was grown in BGMY media for $48 \mathrm{hrs}$ and then transferred to induction media (BMMY) with continuous induction with $1 \%$ absolute methanol for every $12 \mathrm{hrs}$. The supernatant samples were collected at 12 (L1), 24 (L2), 36 (L3), 48 (L4), 60 (L5), and 72 hrs (L6). The high molecular weight of oFGH multimers (approximately $70 \mathrm{kDa}$ ) was observed when the samples were applied for SDS-PAGE without denaturing conditions (heating and reducing by beta-mercaptoethanol (L1-L6)). However, after applying the denaturing conditions only monomer molecules of oFGH were observed at $22 \mathrm{kDa}$ (L7), whilst reducing the samples with beta-mercaptoethanol without heating led to detection of both oFGH monomer $(22 \mathrm{kDa})$ and multimers $(70 \mathrm{kDa})(\mathrm{L} 8)$.

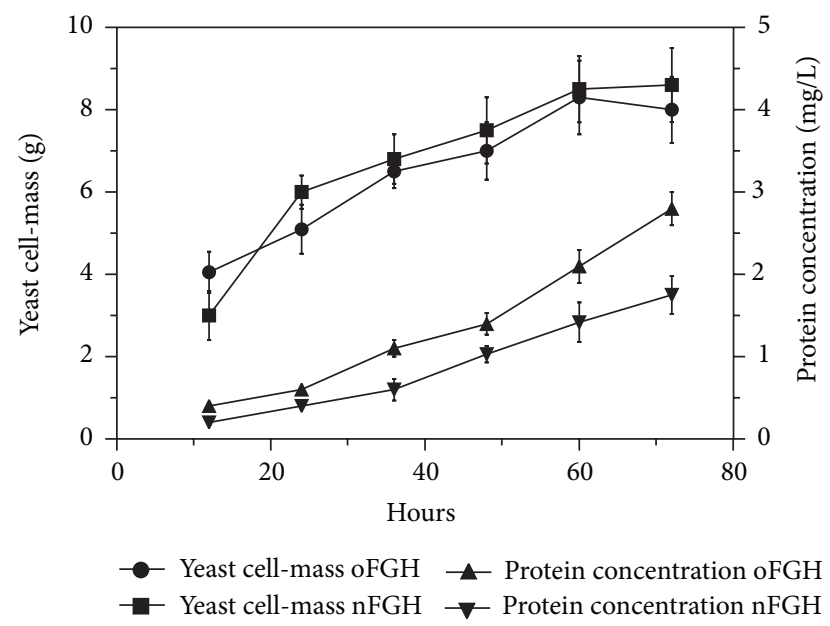

FIgURE 4: Production of recombinant oFGH and nFGH in Pichia pastoris. The yeast was cultured for $72 \mathrm{hrs}$ with induction each $12 \mathrm{hrs}$ with $1 \%$ absolute methanol. The supernatant samples were collected at $12,24,36,48,60$, and $72 \mathrm{hrs}$ and recombinant protein concentration was measured by FGH ELISA. The results showed that the production of oFGH was significantly higher $(P<0.05)$ than $\mathrm{nFGH}$ in one litre culture whereas the total yeast cell-mass was approximately similar (two-tailed paired $t$-test).

pool $[16,17]$ which ultimately could lead to increasing the recombinant protein quantity [18-20]. Therefore, significant increase was observed in the body weight of tiger grouper fingerlings fed with oFGH at third and fourth weeks of the experiment period compared to those fed with nFGH (Figure 5). This increase in the body weight probably was due to the higher concentration of oFGH compared to $\mathrm{nFGH}$ in the culture supernatant $(2.80 \pm 0.27 \mathrm{mg}$ versus $1.75 \pm 0.25 \mathrm{mg}$, resp.). The main outcome of the high content of oFGH in

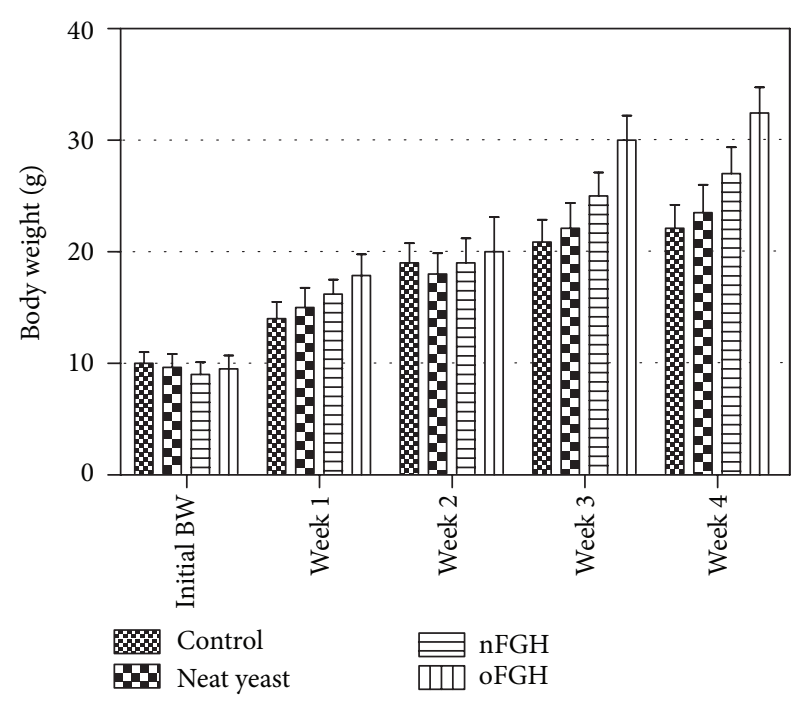

FIGURE 5: The body weight mean of fish fed with culture supernatant from recombinant $P$. pastoris producing FGH. The results showed that tiger grouper fingerlings group fed with oFGH have significantly higher body weight compared to those fed with nFGH at week $3(P<0.05)$ and week $4(P<0.01)$. Both groups fed with nFGH or oFGH showed significant higher body weight compared to neat yeast and control groups at weeks 3 and $4(P<0.05$, nFGH; and $P<0.01$, oFGH) (two-way ANOVA with bonferroni posttest).

the culture supernatant is enhancing the bioavailability of FGH that essentially stimulates fish appetite as well as feed conversion rate $[21,22]$.

It has been known that the administration of FGH enhances many aspects of immune functions including nonspecific defences and cytotoxic, phagocytic, haemolytic, and lysozyme activities and activates immunoglobulin production as a specific defence [23]. Interestingly, it has been found that the culture supernatant containing truncated fish growth hormone has a stronger effect over growth and immune system than cells lysate containing intact growth hormone expressed in P. pastoris [24]. These observations are concomitant with our finding that the final biomass and fish survival percentage were significantly enhanced after feeding with culture supernatant from recombinant $P$. pastoris producing FGHs (Figure 6). In conclusion, the results of this study showed practical and cost-effective administration method of using FGH in mass-scale aquaculture through increasing the expression level of rFGH in yeast supernatant. Further studies are required for practical application in aquaculture farms.

\section{Abbreviations}

FGH: Fish growth hormone

nFGH: Native fish growth hormone protein without codon optimization

oFGH: Fish growth hormone protein after codon optimization

rFGH: Recombinant fish growth hormone

AOX1: Alcohol oxidase gene. 

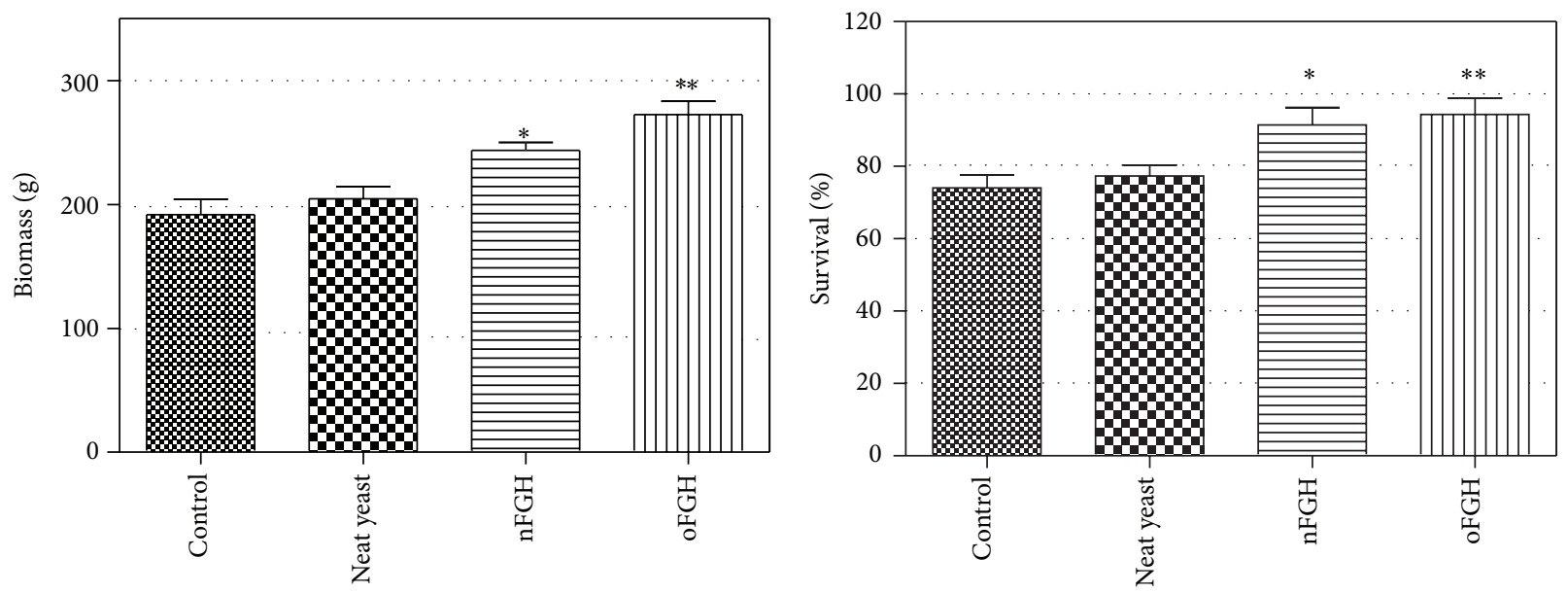

FIGURE 6: The effect of recombinant FGHs on total biomass and fish survival percentage. The results showed that total biomass (total group weight) of tiger grouper fingerlings fed with recombinant oFGH was significantly $(P<0.01)$ higher than those fed with recombinant $\mathrm{nFGH}$, and both FGH groups were significantly higher than those in neat yeast group and control group $(P<0.05, \mathrm{nFGH} ; P<0.01, \mathrm{oFGH})$. The survival percentage of fingerlings in $\mathrm{nFGH}$ - and oFGH-feeding groups was higher $(P<0.05)$ than those in neat yeast and control groups. However, there was no significant difference between nFGH and oFGH groups (one-way ANOVA test).

\section{Highlights}

Fish growth hormone (FGH) has been used as a supplement in fish feed to influence growth and development of farmed fish. FGH can be produced in vitro using molecular biology techniques, and, while many successes have been reported, several problems pertaining to the quantity and quality of the recombinant FGH still exist. In this study, a significant improvement in yield and function of recombinant FGH was achieved in Pichia pastoris using a recombinant gene that had been codon-optimized and expressed through the secretory pathway. This improvement should be useful for consideration in major-scale production of recombinant FGH intended for fish farming.

\section{Conflict of Interests}

The authors declare that there is no conflict of interests regarding the publication of this paper.

\section{Acknowledgment}

This study was sponsored by the Ministry of Higher Education, Malaysia, through the University of Malaya (Fundamental Research Grant Scheme FP051-2010B).

\section{References}

[1] A. Farmanfarmaian and L.-Z. Sun, "Growth hormone effects on essential amino acid absorption, muscle amino acid profile, $\mathrm{N}$ retention and nutritional requirements of striped bass hybrids," Genetic Analysis: Biomolecular Engineering, vol. 15, no. 3-5, pp. 107-113, 1999.

[2] J. T. Silverstein, W. R. Wolters, M. Shimizu, and W. W. Dickhoff, "Bovine growth hormone treatment of channel catfish: Strain and temperature effects on growth, plasma IGF-I levels, feed intake and efficiency and body composition," Aquaculture, vol. 190, no. 1-2, pp. 77-88, 2000.

[3] J. A. Gill, J. P. Sumpter, and E. M. Donaldson, "Recombinant chicken and bovine growth hormones accelerate growth in aquacultured juvenile Pacific salmon Oncorhynchus kisutch," Bio/Technology, vol. 3, no. 7, pp. 643-646, 1985.

[4] S. Moriyama, H. Yamamoto, S. Sugimoto, T. Abe, T. Hirano, and H. Kawauchi, "Oral administration of recombinant salmon growth hormone to rainbow trout, Oncorhynchus mykiss," Aquaculture, vol. 112, no. 1, pp. 99-106, 1993.

[5] Y. H. Chan, C. H. K. Cheng, and K. M. Chan, "Recombinant goldfish growth hormones (gfGH-I and -II) expressed in Escherichia coli have similar biological activities," Comparative Biochemistry and Physiology A: Molecular \& Integrative Physiology, vol. 135, no. 4, pp. 613-624, 2003.

[6] E. de Bernardez Clark, "Refolding of recombinant proteins," Current Opinion in Biotechnology, vol. 9, no. 2, pp. 157-163, 1998.

[7] K. Friehs and K. F. Reardon, "Parameters influencing the productivity of recombinant E. coli cultivations," Advances in Biochemical Engineering/Biotechnology, vol. 48, pp. 53-77, 1993.

[8] J. J. Bai, J. Ma, Q. Jian, X. H. Li, and J. R. Luo, "Clone of cDNA for common carp GH and its expression in prokaryocyte," Chinese Journal of Biochemistry and Molecular Biology, vol. 15, pp. 409$412,1999$.

[9] M. Jin, B. Junjie, L. Xinhui, L. Jianren, J. Qing, and Z. Hongjun, "Expression of rainbow trout growth hormone cDNA in Saccharomyces cerevisiae," Chinese Journal of Biotechnology, vol. 15, no. 4, pp. 219-224, 1999.

[10] H. T. Wang, Y. L. Xu, P. J. Zhang, Y. S. Li, and S. Y. Zuo, "Effects of recombinant yeast on the growth and concentration of hormone in the serum of flounder, Paralichthys olivaceus," Oceanologia et Limnologia Sinica, vol. 31, pp. 135-138, 2000.

[11] D. Chen, F. Yang, W. Wang, and X. Xu, "Intracellular expression of lateolabrox japonicus growth hormone in methyltrophic yeast, Pichia postoris," Progress in Biochemistry and Biophysics, vol. 25, no. 2, pp. 140-143, 1998. 
[12] M. Romanos, "Advances in the use of Pichia pastoris for highlevel gene expression," Current Opinion in Biotechnology, vol. 6, no. 5, pp. 527-533, 1995.

[13] Y. H. Li, J. J. Bai, X. H. Li et al., "Expression of common carp growth hormone in yeast P. pastoris," Chinese Journal of Biochemistry and Molecular Biology, vol. 17, pp. 488-491, 2001.

[14] K. A. Barr, S. A. Hopkins, and K. Sreekrishna, "Protocol for efficient secretion of HAS developed from P. pastoris," PharmEng, vol. 12, pp. 48-51, 1992.

[15] H. A. Rothan, S. H. Teh, K. Haron, and Z. Mohamed, "A comparative study on the expression, purification and functional characterization of human adiponectin in Pichia pastoris and Escherichia coli," International Journal of Molecular Sciences, vol. 13, no. 3, pp. 3549-3562, 2012.

[16] P. Puigbò, E. Guzmán, A. Romeu, and S. Garcia-Vallvé, "OPTIMIZER: a web server for optimizing the codon usage of DNA sequences," Nucleic Acids Research, vol. 35, no. 2, pp. W126W131, 2007.

[17] C. Kurland and J. Gallant, "Errors of heterologous protein expression," Current Opinion in Biotechnology, vol. 7, no. 5, pp. 489-493, 1996.

[18] C. Kimchi-Sarfaty, J. M. Oh, I. W. Kim et al., "A "silent" polymorphism in the MDR1 gene changes substrate specificity," Science, vol. 315, no. 5811, pp. 525-528, 2007.

[19] E. Angov, C. J. Hillier, R. L. Kincaid, and J. A. Lyon, "Heterologous protein expression is enhanced by harmonizing the codon usage frequencies of the target gene with those of the expression host," PLoS ONE, vol. 3, no. 5, Article ID e2189, 2008.

[20] M. Marin, "Folding at the rhythm of the rare codon beat," Biotechnology Journal, vol. 3, no. 8, pp. 1047-1057, 2008.

[21] J. I. Johnsson and B. T. Bjornsson, "Growth hormone increases growth rate, appetite and dominance in juvenile rainbow trout, Oncorhynchus mykiss," Animal Behaviour, vol. 48, no. 1, pp. 177186, 1994.

[22] B. T. Björnsson, "The biology of salmon growth hormone: from daylight to dominance," Fish Physiology and Biochemistry, vol. 17, no. 1-6, pp. 9-24, 1997.

[23] T. Yada, "Growth hormone and fish immune system," General and Comparative Endocrinology, vol. 152, no. 2-3, pp. 353-358, 2007.

[24] J. Acosta, Y. Carpio, V. Besada et al., "Recombinant truncated tilapia growth hormone enhances growth and innate immunity in tilapia fry (Oreochromis sp.)," General and Comparative Endocrinology, vol. 157, no. 1, pp. 49-57, 2008. 

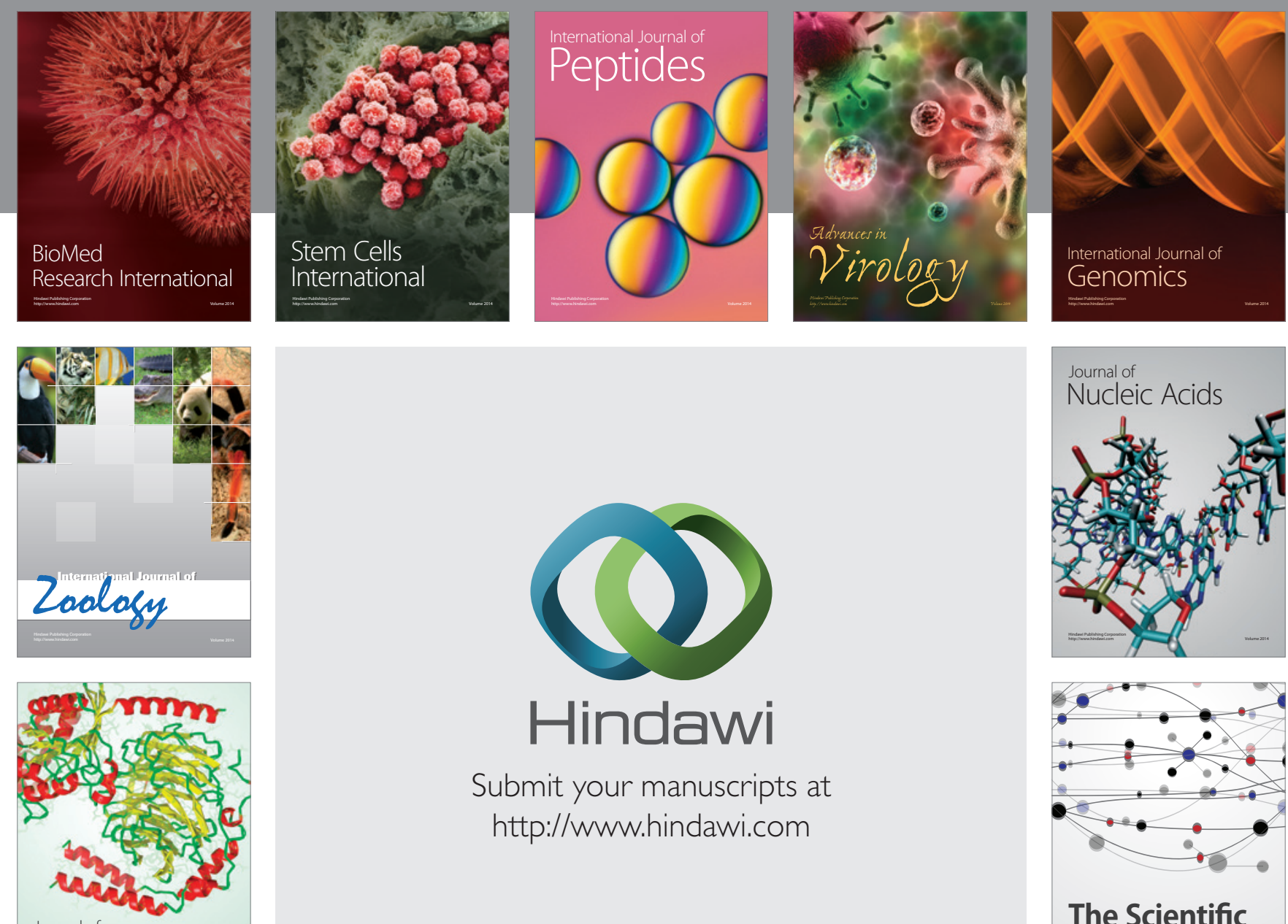

Submit your manuscripts at

http://www.hindawi.com

Journal of
Signal Transduction
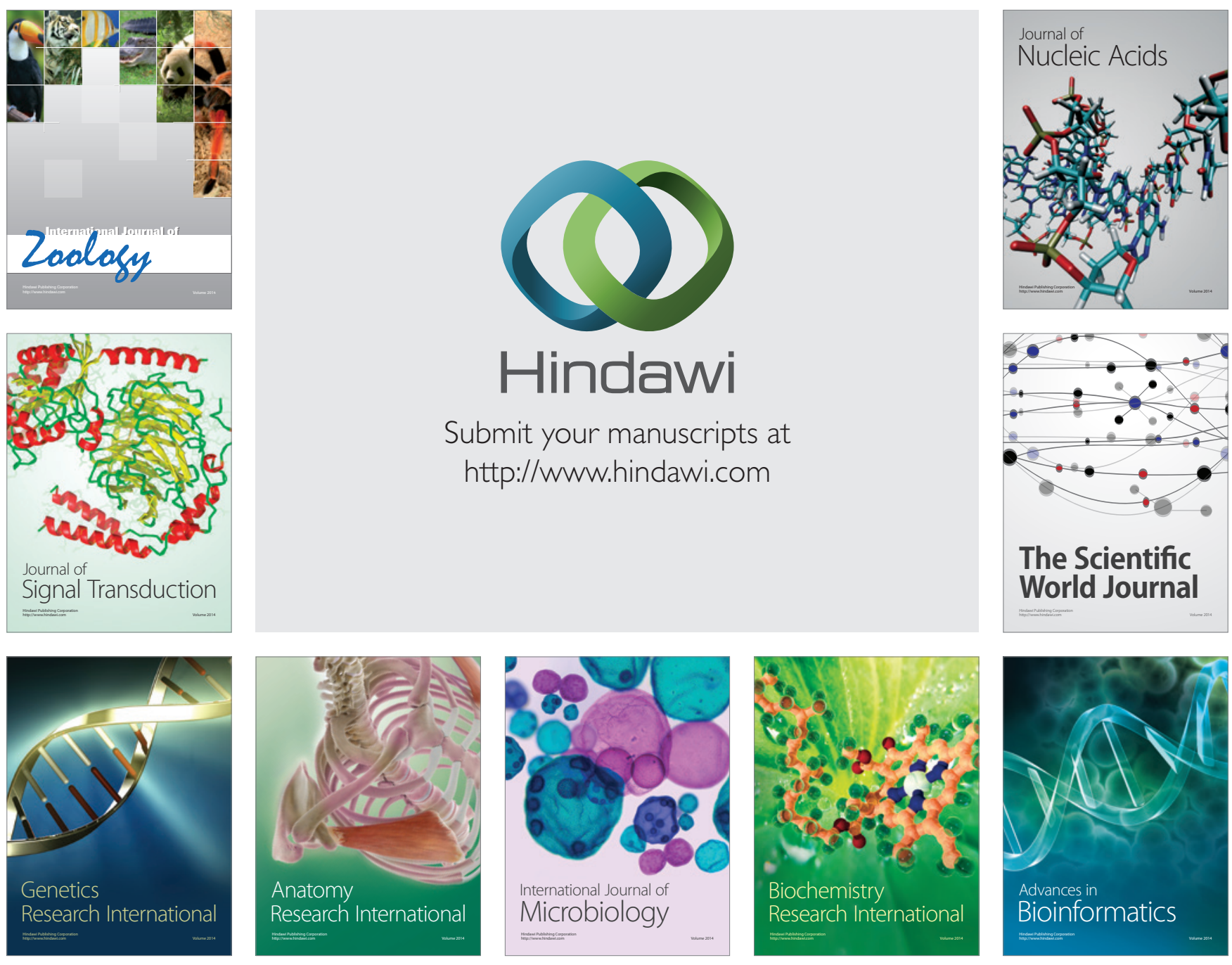

The Scientific World Journal
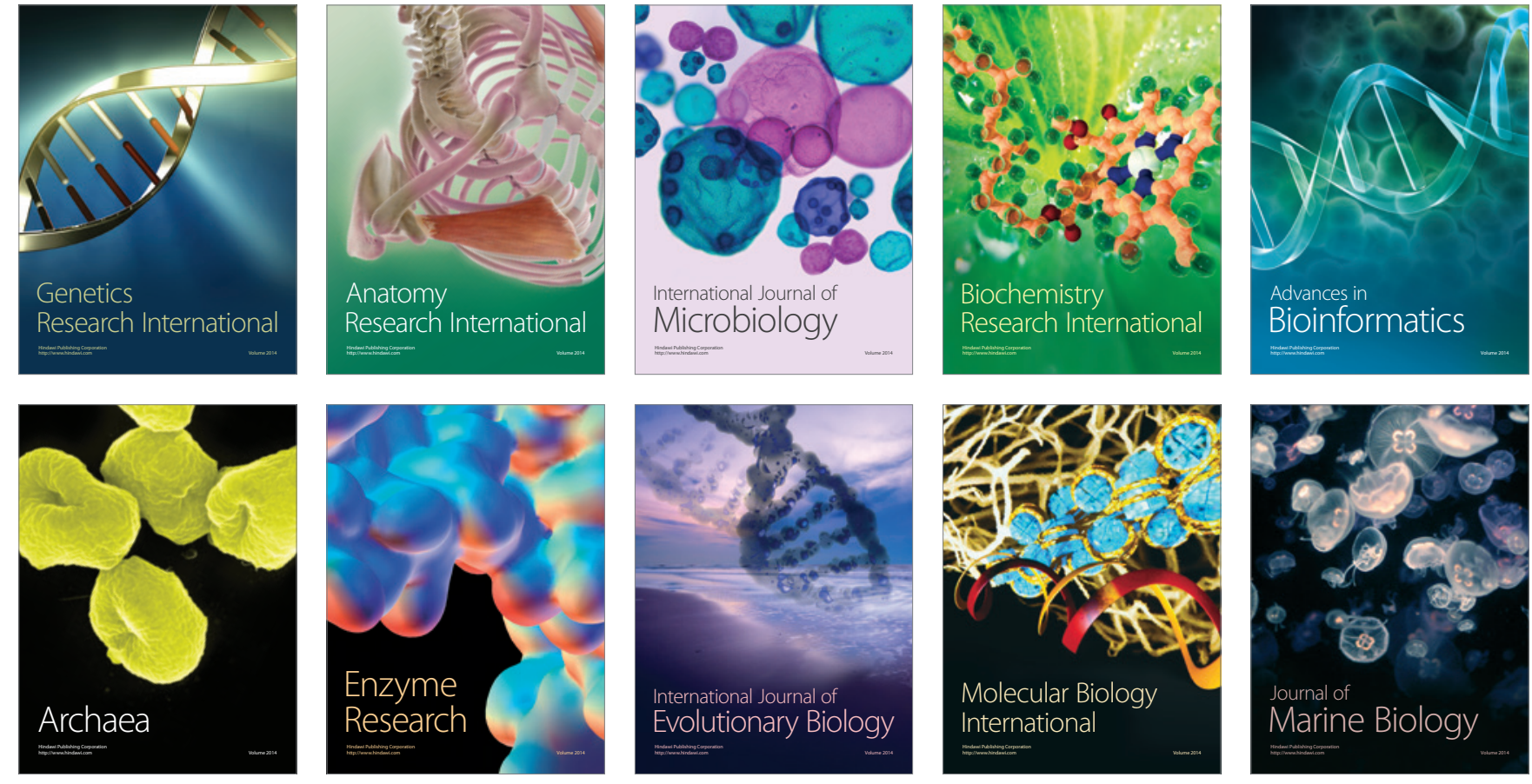